\title{
In vitro and in vivo anticancer effects of mevalonate pathway modulation on human cancer cells
}

\author{
P Jiang ${ }^{*}, 1$, R Mukthavaram ${ }^{1}$, Y Chao ${ }^{1}$, N Nomura ${ }^{1}$, I S Bharati ${ }^{1}$, V Fogal ${ }^{1}$, S Pastorino ${ }^{1}$, D Teng ${ }^{2}$, X Cong $^{1}$, \\ S C Pingle ${ }^{1}$, S Kapoor ${ }^{3}, \mathrm{~K}$ Shetty ${ }^{3}, \mathrm{~A} \mathrm{Aggrawal}^{3}, \mathrm{~S} \mathrm{Vali}^{3}, \mathrm{~T} \mathrm{Abbasi}^{3}, \mathrm{~S} \mathrm{Chien}^{2}$ and S Kesari ${ }^{\star 1,4}$ \\ ${ }^{1}$ Translational Neuro-Oncology Laboratories, Moores Cancer Center, UC San Diego, La Jolla, CA 92093, USA; ${ }^{2}$ Departments of \\ Bioengineering and Medicine and Institute of Engineering in Medicine, UC San Diego, La Jolla, CA 92093, USA; ${ }^{3}$ Cellworks Group, \\ Inc., 2025 Gateway Place, Suite 265, San Jose, CA 95110, USA and ${ }^{4}$ Department of Neurosciences, UC San Diego, La Jolla, CA \\ 92093, USA
}

Background: The increasing usage of statins (the 3-hydroxy-3-methylglutaryl-coenzyme A reductase inhibitors) has revealed a number of unexpected beneficial effects, including a reduction in cancer risk.

Methods: We investigated the direct anticancer effects of different statins approved for clinical use on human breast and brain cancer cells. We also explored the effects of statins on cancer cells using in silico simulations.

\begin{abstract}
Results: In vitro studies showed that cerivastatin, pitavastatin, and fluvastatin were the most potent anti-proliferative, autophagy inducing agents in human cancer cells including stem cell-like primary glioblastoma cell lines. Consistently, pitavastatin was more effective than fluvastatin in inhibiting U87 tumour growth in vivo. Intraperitoneal injection was much better than oral administration in delaying glioblastoma growth. Following statin treatment, tumour cells were rescued by adding mevalonate and geranylgeranyl pyrophosphate. Knockdown of geranylgeranyl pyrophosphate synthetase-1 also induced strong cell autophagy and cell death in vitro and reduced U87 tumour growth in vivo. These data demonstrate that statins main effect is via targeting the mevalonate synthesis pathway in tumour cells.
\end{abstract}

Conclusions: Our study demonstrates the potent anticancer effects of statins. These safe and well-tolerated drugs need to be further investigated as cancer chemotherapeutics in comprehensive clinical studies.

Statins are potent competitive inhibitors of 3-hydroxy-3-methylglutaryl-CoA (HMG-CoA) reductase (HMGCR), a rate-limiting enzyme in the mevalonate pathway (cholesterol synthesis or isoprenoid pathway; Endo et al, 1976; Alberts et al, 1980; Brown and Goldstein, 1986). Statins are clinically used to treat hypercholesterolaemia and reduce the incidence of cardiovascular and cerebrovascular events. As of 2013, there are seven statin drugs available in the US market: atorvastatin (Lipitor and Torvast), fluvastatin (Lescol), lovastatin (Mevacor, Altocor, Altoprev), pitavastatin (Livalo, Pitava), pravastatin (Pravachol, Selektine, Lipostat), rosuvastatin (Crestor), and simvastatin (Zocor,
Lipex)(http://www.fda.gov/Drugs/DrugSafety/InformationbyDrugClass/ucm294358.htm). Another statin drug, cerivastatin (Baycol, Lipobay), was withdrawn from the market in 2001, due to fatal rhabdomyolysis and kidney failure (Furberg and Pitt, 2001). Clinical trials with mevastatin were performed in the late 1970s in Japan, but it was never marketed (Endo, 2004). These drugs are well tolerated and showed few adverse effects. In the last 20 years, increasing clinical data and epidemiological studies support that statins may have a role in the prevention of cancer. This anticancer effect may be unrelated to the lipid lowering function of statins but the details are still unknown (Tobert, 2003; Clendening and Penn, 
2012). Multiple in vitro and in vivo studies have shown that statins have a wide range of anticancer activities in various cancers, as well as gliomas (Clendening and Penn, 2012; Thurnher et al, 2012). Overall, epidemiological data support the anticancer activity of statins, but the benefits have been mixed and largely depend on the type of tumour, the particular statin used and the end points used in the study.

In the present study, we explored the anticancer effect of nine statins in a highly metastasising cancer (breast cancer) and a highly aggressive cancer (glioblastoma). Our work lays the foundation for further investigations and more rational design for new statinsbased anticancer drugs or as an adjuvant with standard therapies.

\section{MATERIALS AND METHODS}

Chemical compounds. Nine statin drugs were obtained from commercial sources: Atorvastatin calcium salt trihydrate, simvastatin, pravastatin, mevastatin (PZ0001, S6196, P4498, M2537 Sigma, St Louis, MO, USA); fluvastatin, rosuvastatin, cerivastatin (F601250, R700500, C277000, Toronto Research Chemicals Inc., Toronto, ON, Canada); pitavastatin (S1759, Selleckchem, Boston, MA, USA); lovastatin (430-103-M050, Enzo life sciences, Farmingdale, NY, USA). We prepared $10 \mathrm{~mm}$ stock solution in DMSO for in vitro testing. For in vivo testing, pitavastatin was prepared as fresh suspension in methycellulose at $0.4 \mathrm{mg} \mathrm{ml}^{-1}$ (for oral gavage). Fluvastatin, cerivastatin, and pitavastatin were prepared as a solution at $0.25 \mathrm{mg} \mathrm{ml}^{-1}$ in PBS for IP injection. GGTI-298 and FTI-277 (G5169, A1393, Sigma) were made into $10 \mathrm{~mm}$ stock solution in DMSO. AICAR (\#S1802, Selleckchem) was made into $100 \mathrm{~mm}$ stock solution in PBS. Intermediate products of cholesterol synthesis were purchased from Sigma: mevalonolactone (M4667), farnesyl pyrophosphate ammonium salt (F6892), geranylgeranyl pyrophosphate ammonium salt (G6025), squalene (S3626), cholesterol solution (S5442), geranylpyrophosphate (G6772), and isopentenyl pyrophosphate triammonium salt solution (I0503).

Cell cultures. Established breast cancer (MDA-MB-231, MDAMB-432, MDA-MB-435) and glioblastoma (GBM) cell lines (A172, LN443, U87, U118, U251) were grown in DMEM medium supplemented with $10 \% \mathrm{FBS}$ at $37^{\circ} \mathrm{C}$ in a humidified $5 \% \mathrm{CO}_{2}$ atmosphere. Patient-derived stem cell-like GBM cell lines (GBM4, GBM8, BT70, BT74, SK115) were cultured in complete NeuroCult Proliferation Medium with hEGF and hFGF (\#05702, \#02653, and \#02654, Stemcell Technologies, Vancouver, BC, Canada).

Cellular proliferation/viability inhibition assay. Cells were seeded into 96 -well plates at a density of 2000 cells per well. The statin drugs were serially diluted using the culture media and cells were incubated for the times mentioned. Alamar Blue (\#BUF012B, AbDSerotec, Kidlington, UK) was then added, according to the manufacturer's manual, directly to the culture medium and the fluorescence signal was read at 560/590 after $4-16 \mathrm{~h}$ to determine the number of viable cells (Infinite M200, Tecan Group Ltd, Männedorf, Switzerland). The $\mathrm{IC}_{50}$ values were calculated using commercial software (Prism, Graphpad Software, La Jolla, CA, USA).

Cell growth inhibition assay. Tumour cells were added to six-well plates overnight at a density of $5 \times 10^{4}$ per well and then treated with 1,3 and $10 \mu \mathrm{M}$ pitavastatin or vehicle (DMSO). From day 1 to day 4 after treatment, the cell number in each well was counted by trypan blue assays according to standard protocols. All experiments were repeated in triplets.

Cell cycle analysis. Tumour cells were plated at a density of $5 \times 10^{5}$ cells in $6-\mathrm{cm}$ dishes overnight. Cells were washed twice with PBS, followed by fresh media with pitavastatin. After $24 \mathrm{~h}$, the cells were fixed in $70 \%$ ethanol in PBS and stored at $-20^{\circ} \mathrm{C}$ for 24h. Propidium iodide staining of DNA was carried out and analysed via flow cytometry (Canto, BD FACS) to determine the distribution of cells in different phases of the cell cycle with the aid of ModFit LT software (version 3.0).

Cellular autophagy detection. We confirmed that statin induced autophagy by two commonly used assays: quantification of GFPLC3 puncta and measuring LC3-II protein. LC3 (microtubule-associated protein 1 light chain 3) is disturbed evenly throughout the cytoplasm under normal condition, but were recruited to autophagosome membrane. Green fluorescent protein fused with LC3 (GFPLC3) can be visualised when recruited to autophagosome membrane as punctuate distribution following statin treatment. During cell autophagy, the cytosolic form of LC3-1 $(16-18 \mathrm{kDa})$ is lapidated and transformed to LC3-II $(14-16 \mathrm{kDa})$, and it can be separated on SDS-PAGE gel.

Quantifying GFPLC3 puncta by fluorescence microscopy. Stable U251 and MDA-MB-435 cell lines expressing GFPLC3 (GFP fusion with LC3) or GFP (retroviral transduction) were selected. U251/GFPLC3 cells or U251/GFP cells were seeded to $5 \times 10^{4}$ per well in 24-well plates, and pitavastatin was added to final concentrations of $0.1 \mu \mathrm{M}$ for U251 and $0.5 \mu \mathrm{M}$ for MDA-MB-435 cells. The cells were visualised at $12,24,36,48$, and $60 \mathrm{~h}$. Fluorescent images were taken at five random locations in each well. The GFPLC3 clusters were quantified using the particle counting function in Metamorph software (Molecular Devices LLC, Sunnyvale, CA, USA). The number of cells and the GFPLC3 cluster in the cells were quantified using Cell Profiler software. The number of LC3 clusters of an image was normalised using the number of cells in that image. The mean and standard error of the mean of the five images ( $>100$ cells) were calculated.

Conversion of LC3-I to LC3-II by western blotting. U87 cell were treated with $1 \mu \mathrm{M}$ pitavastatin for 48,72 , and $96 \mathrm{~h}$, the cell lysates were harvested, and $10 \mu \mathrm{g}$ whole protein was loaded onto $14 \%$ SDS-PAGE gel. The GBM4, GBM8, BT70, and BT74 sphere cells were treated with $0.3 \mu \mathrm{M}$ pitavastatin for $72 \mathrm{~h}$. After transferring the proteins to PVDF membrane, the following antibodies were used for immunoblotting: LC3 (NB100-2220, Novus Biologicals, Cambridge, UK) and tubulin (T4026, Sigma). The resultant protein bands were visualised after incubation with HRP-labelled secondary antibodies, and reacted with substrates of a supersignal kit (\#1856136, Thermo Scientific, Waltham, MA, USA). The results were recorded on autoradiography film (\#1651496, Kodak, Rochester, NY, USA), and the films were scanned after development. The ratios of band intensities of LC3-II/LC3-I were calculated by NIH Image-J software.

Antitumour studies in vivo. We evaluated the antitumour efficacy of pitavastatin in xenografts mouse model by two types of treatments: gavage and intraperitoneal (i.p.) injection. Fluvastatin was tested by injection (i.p.) at the same dose. Six-to-eightweeks nude (nu/nu) mice were obtained from the UCSD breeding facility. We engrafted $2 \times 10^{6}$ U87 cells at the left and right flanks of the mice. Tumour size was measured every 2 days from day 6 , and mice with similar tumour sizes were divided into two groups at day 10. After 10 days of tumour growth, the mice were treated by oral gavage with $8 \mathrm{mg} \mathrm{kg}^{-1}$ once per day on a 5-days-on, 2-daysoff schedule for 2 weeks, and $8 \mathrm{mg} \mathrm{kg}^{-1}$ twice per day thereafter. The control group was treated with the same amount of methycellulose suspension without the drug. For i.p. injection, the mice were treated with $1 \mathrm{mg} \mathrm{kg}^{-1}$ once per day, the control group was treated with PBS. Tumour measurements were done every 2 days and all mice were euthanized after the tumour reached a size of more than $1 \mathrm{~cm}$ or showed necrosis according to our animal protocol. The tumours were dissected and weighed, and 
then the typical tumours in each group were fixed with $10 \%$ formalin, and paraffin slides were stained with H\&E and Ki67 antibody.

All animal studies were conducted in the veterinary facilities of Moores Cancer Center in accordance to institutional rules, state and federal laws, and ethical guidelines for experimental animal care.

Cell rescue after statins treatments. The U87 cells were seeded onto 24 -well plates at $4 \times 10^{4} \mathrm{ml}^{-1}$ overnight; then $10 \mu \mathrm{M}$ pitavastatin was added. The intermediate products in the cholesterol synthesis pathway were added with the statins (Brown and Goldstein, 1986; Wong et al, 2002). The final concentration was $100 \mu \mathrm{M}$ for mevalonate, and $10 \mu \mathrm{M}$ for GPP, IPP, GGPP, FPP, and squalena. Cholesterol solution was diluted to $5 \times$. Pictures were taken daily and cell viability tested after 3 days of treatment by alamar blue assay as mentioned above.

GGPPS-1 knockdown by shRNA. 293T cells were cultured to $70 \%$ confluent and transfected with pCMV-VSVG, pCMV-dR8.9 packaging plasmids with SHC002 (pKLO.1 nontarget control plasmid, Sigma SHC002), or pKLO.1- $\triangle$ GGPS1 shRNA1 and 2 (TRCN00000045788 and TRCN00000045790). After 48 and $72 \mathrm{~h}$, the lentivirus suspension was collected and used to infect U87 cells. Cells were observed using a light microscope daily. The collected cells with infected control or knockdown shRNA at $72 \mathrm{~h}$ had no significant morphological changes. The GGPPS-1 protein expression was confirmed to decrease at $72 \mathrm{~h}$ after infection. After 3 days of infection, the cells were collected, and $1 \times 10^{6}$ U87 SHC2 cells and GGPPS-1 knockdown cells were engrafted at the right and left flanks, respectively, in the same six mice. We measured the tumour sizes weekly during the experiment and weighed the individual tumours after the killing.

Simulation-based analysis. Predictive computational studies testing the effect of statins were performed using in silico simulations from Cellworks Group, Inc (version 8.6; Saratoga, CA, USA). This model (Pingle et al, 2014) represents signalling and metabolic pathways in cancer physiology that integrates all cancer phenotypes such as proliferation, apoptosis, viability, angiogenesis, tumour metabolism, and metastasis. The details of these assay are shown in the online Supplementary Material.

Statistical analysis. The statistical significance of differences was determined by Student's $t$-test. $P<0.05$ was considered as a statistically significant difference. The number of asterisks indicates the statistical significance level: one asterisk $\left({ }^{*}\right)$ for 0.05 , two $\left(^{* *}\right)$ for 0.01 , and three $\left(^{* *}\right)$ for 0.001 in bar graphs. Data of $P>0.05$ are considered not significant and labelled \#.

\section{RESULTS}

Statins inhibit proliferation/viability of human tumour cell lines. To evaluate the anticancer effects of statins, we tested the anti-proliferative/viability capability of the nine statin drugs in vitro using GBM and breast cancer cell lines and calculated the $\mathrm{IC}_{50}$. As expected, statins showed anti-proliferative/viability activity in almost all cell lines with $\mathrm{IC}_{50}$ values in the following order: cerivastatin $<$ pitavastatin $<$ fluvastatin $<$ simuvastatin $<$ atorvastatin/lovastatin/mevastatin $<$ rosuvastatin $<$ pravastatin (Figure $1 \mathrm{~A}$ and Supplementary Figure S1A). In A172 cells, the $\mathrm{IC}_{50}$ values for the three most potent statins, cerivastatin, pitavastatin, and fluvastatin were $0.098,0.334$, and $0.922 \mu \mathrm{M}$ respectively. The results were similar in all three GBM (A172, U87, U251) and three breast cancer cell lines (MDA-MB-231, -432, and -435). As shown in Supplementary Figure S1C, the anti-proliferative/viability effect was, in part, due to the decrease in cell number after statin treatment. We also confirmed that these statins are potent inhibitors of stem cell-like GBM cells isolated from patients and cultured in non-serum medium (Figure 1A and Supplementary Figure S1B). Importantly, following longer treatment duration, there was an enhanced anti-proliferative/viability effect (Supplementary Figure S2): for example, the $\mathrm{IC}_{50}$ of pitavastatin in U87 cells was 21.2, 7.30, and $4.80 \mu \mathrm{M}$ for 2-, 3- and 4-day treatments, respectively. This enhancement is independent of cell lines as all five GBM cell lines tested and the two statins tested demonstrated similar results. We also found that the anticancer effect of statins was dependent on the specific cell lines: U87 was the most resistant and LN443 was most sensitive at all time points tested for pitavastatin and cerivastatin, according to their $\mathrm{IC}_{50}$ values in all five cell lines (Supplementary Figure S2). These data suggest that although some statins can inhibit tumour cell growth by interfering with critical metabolic pathways that are conserved in all cells, the genetic background of tumour cells strongly influences drug response.

The in silico simulation-based studies independently confirmed that statins inhibited GBM cells (A172) and breast cancer cells (MDA-MB-231) by downregulating all the cell proliferation markers (Supplementary Figure S3). The effect of statins on other key kinases such as RAS, ERK, and PKC is also shown in Supplementary Figure S4.

As a standard treatment for GBM patient, radiotherapy has been shown to have promising efficacy. Statins co-treated with 2, 5, and 10 Gy irradiation did not show significant antagonistic effect in the inhibition of U87 cell line growth as the $\mathrm{IC}_{50}$ slightly increased compare with statin alone (Supplementary Figure S5).

Statins induce cell cycle arrest in tumour cells. We analysed the effects of statins on cell cycle in tumour cells. Statins induced G1/S growth arrest in GBM cell lines (A172, U87, and U251) and breast cancer cell lines (MDA-MB-231, 432, 435). In Table 1, we show that pitavastatin triggers cell cycle arrest as early as $12 \mathrm{~h}$ after treatment; tumour cells in the S phase dramatically decreased, whereas the cell population in the G0/G1 phase increased. This result is consistent with previous reports on lovastatin (Jakobisiak et al, 1991; Keyomarsi et al, 1991). We observed that statins induced overexpression of cyclin-dependant kinase p21 in MDAMB-431, U87 and GBM4 cells, but this may be unrelated to p53 activity, as p53 protein was not detected in U87 and GBM4 cells but under the same conditions p53 was detected in MDA-MB-431 (Figure 1C).

The precise mechanism of molecular modulation of cell cycle arrest remains ill defined, but it is important to understand and to predict tumour cell response to statins. To address these questions, we used in silico simulation to run predictive studies and analysis of the intermediate mechanisms. Our simulation-based assays indicate that sensitivity of U87 cells to statins increases with p53 mutation, p21 deletion, and CDK4 mutations. In contrast, mutations of wild-type p16 and PTEN increase resistance of U87 cells to statins (Supplementary Figure S6). Each of these mutations causes an upregulation of the mevalonate pathway, making the cells more sensitive to inhibition of HMGCR. Our simulation data may explain the scientific rationale behind the cell death caused by statins in GBM cells.

Statins induce cellular autophagy. Previous studies have reported that statins can trigger a subset of tumour cells to undergo apoptosis (Jones et al, 1994; Dimitroulakos et al, 1999). In our previous study (Jiang et al, 2014), we observed strong cellular autophagy but did not detect apoptotic signals in GBM cells treated with pitavastatin. We explored all three potent statins and found that all of them induce autophagy (Figure 2A). To quantitate this effect, we used the GFPLC3 aggregation dot assay. In GBM cell lines, the GFPLC3 dots significantly increased at $24 \mathrm{~h}$, but for MDA-MB-432 cells it required $36 \mathrm{~h}$ (Figure 2B). After 2 days of treatment, LC3-II isoform increased dramatically (Figure 2C). 

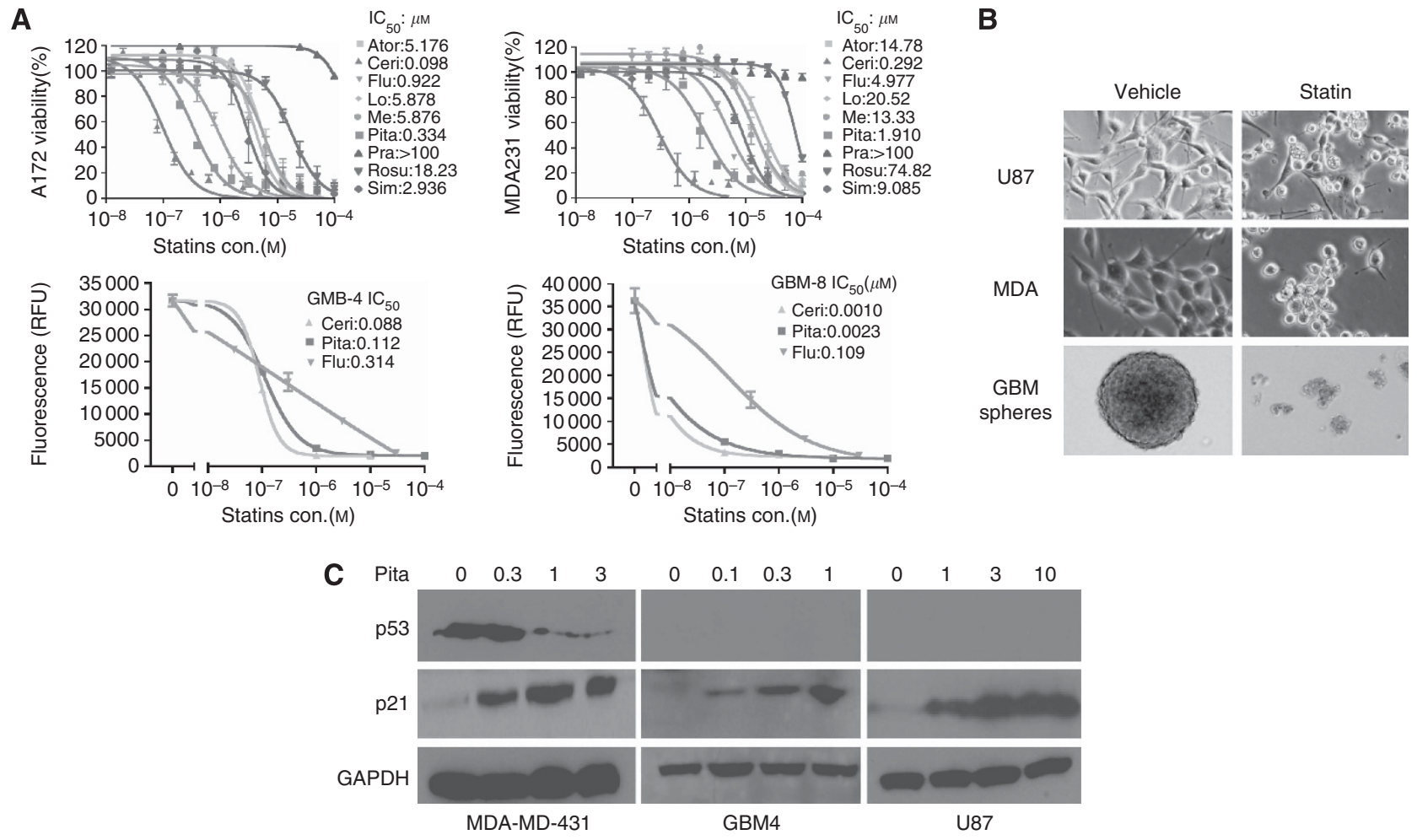

Figure 1. Statins are potent inhibitors for human cancer cells. (A) Comparison of $I C_{50}$ values of statins for breast cancer cell lines (MDA-MB-231) and GBM cell lines (A172). The most potent statins are cerivastatin, pitavastatin, and fluvastatin according to the $I C_{50}$ values in all cell lines. Primary GBM cells (GBM4, GBM8) were also sensitive to statin treatment; cerivastatin, pitavastatin, and fluvastatin were potent cancer cell growth inhibitors, with $\mathrm{IC}_{50}$ values ranging from $1 \mathrm{~nm}$ to $1.5 \mu \mathrm{M}$. (B) Morphological changes of MDA-MB-432, U87 and GBM spheres after statin treatment. After 24-h treatments, tumour cells morphology showed rounding and detachment (U87 and MDA-MB-432 cells). (C) Statins induced p21 expression and p53 status. After pitavastatin treatment, p21 increased related with drug dose (0.3-3 $\mu \mathrm{M}$ for MDA-MB-431, 0.1-1 $\mu \mathrm{M}$ for GBM4 and 1-10 $\mu \mathrm{M}$ for U87). For breast cancer cell line, p53 decreased after treatment; but for GBM4 and U87 cell line, no p53 signal was observed. Abbreviations: Ator = atorvastatin; Ceri = cerivastatin; Flu = fluvastatin; Lo = lovastatin; Me = mevastatin; Pita = pitavastatin; Pra = pravastatin; Rosu = rosuvastatin; $\operatorname{Sim}=$ simvastatin.

Table 1. After statin treatment, breast cancer cells and glioblastoma (GBM) cells strongly arrested in G0/G1 phase and dramatically depleted cells at $\mathrm{S}$ phase

\begin{tabular}{|l|c|l|c|c|c|}
\hline Cell & Time & Treatment & G1/G0 & S & G2/M \\
\hline U87 & $12 \mathrm{~h}$ & Vehicle & 55.20 & 33.03 & 11.70 \\
& & Pita & 67.42 & 18.58 & 13.90 \\
\hline & $24 \mathrm{~h}$ & Vehicle & 65.91 & 21.41 & 12.67 \\
& & Pita & 74.58 & 12.89 & 12.53 \\
\hline & \multirow{2}{*}{$\mathrm{h} h$} & Vehicle & 74.88 & 17.27 & 7.85 \\
& & Pita & 90.21 & 5.71 & 4.09 \\
\hline MDA-MB-431 & \multirow{2}{*}{$\mathrm{h}$} & Vehicle & 49.42 & 23.8 & 26.78 \\
& & Pita & 51.51 & 18.92 & 25.56 \\
\hline & $24 \mathrm{~h}$ & Vehicle & 49.82 & 41.38 & 8.80 \\
& & Pita & 69.83 & 24.15 & 6.02 \\
\hline & $36 \mathrm{~h}$ & Vehicle & 65.91 & 21.41 & 12.67 \\
& & Pita & 84.47 & 6.69 & 8.85 \\
\hline
\end{tabular}

We also observed autophagy in GBM sphere cultures, as four cell lines cultured and passaged in neural stem cell media (GBM4, GBM8, BT70, BT74) increased the LC3-II/I ratio significantly (Figure 2C). In silico simulation-based predictive data suggest that statins induce autophagy by upregulating ATG9B signal as other key makers analysed were downregulated (Supplementary Figure S4). The effect of statins was also tested on the U251 simulation model. Simulation demonstrated that statins induced autophagy in U251 cells and was associated with an increase in Beclin1,
ATGB13, and autophagosome (Supplementary Figure S7). Thus, these data also suggest that statins may cause cell death by autophagy as the key end point effect.

Tumour cells rescued by intermediates of mevalonate pathway after statin treatment. Statins potently inhibit the mevalonate pathway, which is vital for many biologically active metabolites in addition to producing cholesterol. To determine which products of the mevalonate pathway have a role in the anticancer effect of statin, we attempted to rescue U87 cells by adding intermediates of this pathway. Only mevalonate and GGPP were able to rescue GBM and breast cancer cells after statin treatment; other products such as cholesterol, farnesyl pyrophosphate, geranylpyrophosphate and isopentenyl pyrophosphate had very little or no effect on statin-induced growth inhibition and cell death (Figure 3A and B). We also found that GGPP suppressed cell autophagy induced by statins (Figure 3C). These data indicate that GGPP has a critical role in tumour cell growth and proliferation.

In cells, transfer of a geranylgeranyl moiety to C-terminal cysteine(s) of the target protein is catalysed by geranylgeranyltransferase I or II (Rab geranylgeranyl-transferase). It is interesting that a geranylgeranyltransferase I inhibitor GGTI-298 did not inhibit cell autophagy (Figure 3C). But pitavastatin and GGTI-298 did not show a synergistic effect to inhibit U87 and U251 cell proliferation/viability. At higher concentrations of GGTI-298, such as $5 \mu \mathrm{M}$ (about $2 \mathrm{IC}_{50}$ of GGTI-298), the $\mathrm{IC}_{50}$ of pitavastatin increased further (Supplementary Figures S8 and S9). Similar results were also seen in the predictive simulation-based model. These data indicate that other GGPP transferases such as GGTase 
A

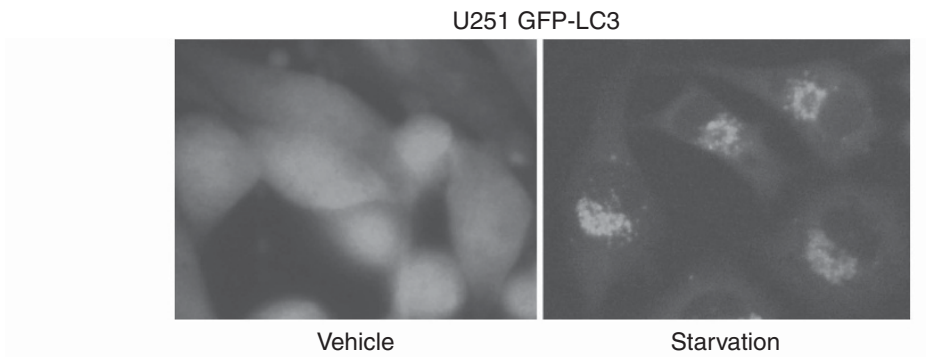

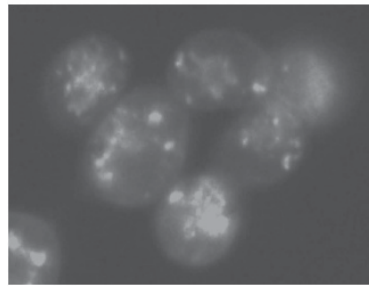

Cerivastatin

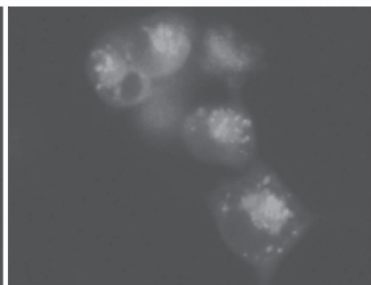

Pitavastatin

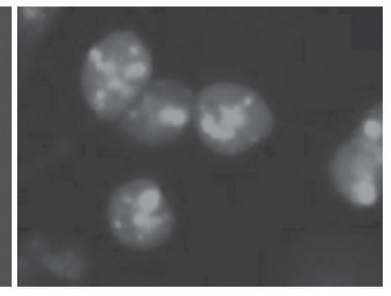

Fluvastatin

B
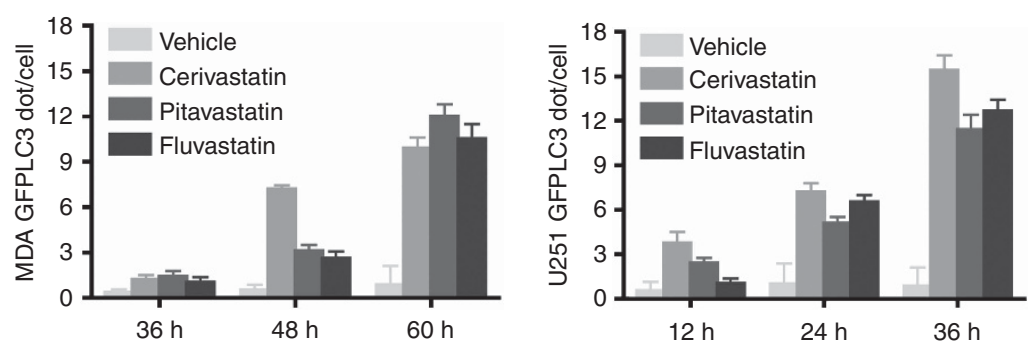

C

U87 cell
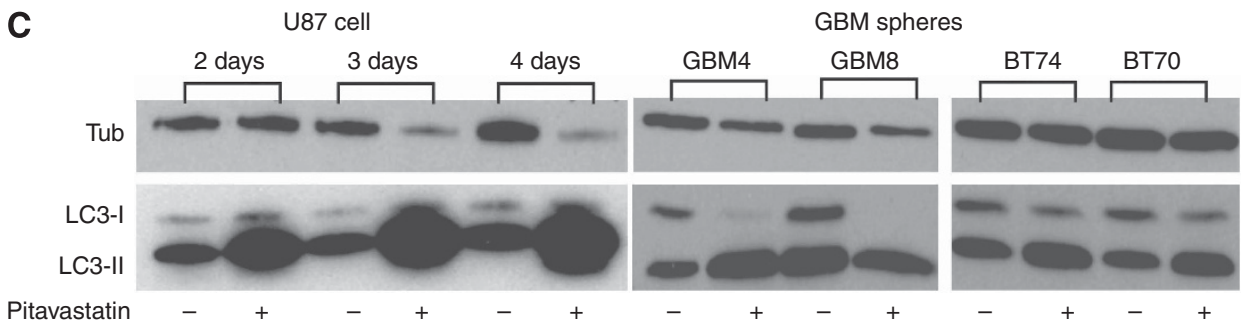

Figure 2. Statins treatment induced cell autophagy. (A) GFPLC3 puncta formed after statin treatment in tumour cell vs the control cell GFPLC3, which were evenly distributed in the cytoplasm. (B) Quantification of GFPLC3 puncta after cerivastatin, pitavastatin, and fluvastatin treatment. The GFPLC3 dot per cell significantly increased after statins treatment in all times (12 h, $24 \mathrm{~h}, 36 \mathrm{~h}$ for U251 cell; $36 \mathrm{~h}, 48 \mathrm{~h}, 60 \mathrm{~h}$ for MDA-MB-435 cell). After statins treatment, average GFPLC3 dot per U251 cell increased from $1.8 \pm 1.3$ to $15.4 \pm 4.6,11.4 \pm 4.6,12.6 \pm 3.5(P=0.070,0.025$ and 0.038 ; $n=100)$ at $36 \mathrm{~h}$; in MDA-MB-435 the average GFPLC3 dot increased from $1.5 \pm 1.2$ to $9.9 \pm 3.2,12.0 \pm 3.2$ and $10.5 \pm 4.4(P=0.085,0.039$ and $0.044 ; n=100$ ) at $60 \mathrm{~h}$. (C) Western blotting showed that in U87 cells treated with pitavastatin, the LC3-II isoform dramatically increased after statin treatment and showed at day 2, 3 and 4. Statin treatment also induced autophagy for GBM stem cell-like cells, the western blot of LC3 showed that the LC3-I isoform decreased and at the same time LC3-II increased, the ratio of LC3-II/LC3-I increased dramatically (2.6 to 25.1 for GBM4; 2.1 to 34.8 for GBM8; 2.3 to 5 for BT74 and 1.6 to 3.3 for BT70.

II/ RabGGTase, instead of the geranylgeranyl-transferase I, may contribute to the functional prenylation of proteins in tumour. Unfortunately no specific GGTase II inhibitor is commercial available, although previous studies have shown that the GGTase II/RabGGTase inhibition has synergistic effect with lovastatin in attenuating the growth of malignant peripheral nerve sheath tumour cells (Sane et al, 2010). The exact molecular mechanisms need to be elucidated, specifically how GGPP, GGTase II/RabGGTase, and the protein substrate(s) of GGTase can affect tumour cell growth and proliferation.

GGPPS-1 knockdown also induced cell death and autophagy. The rescue of tumour cells by GGPP following statin treatment indicates that GGPP has a critical role in cell growth and proliferation. We evaluated whether depletion of GGPP can induce tumour cell death and autophagy. GGPPS-1 is the only known enzyme that synthesizes GGPP from FPP. We knocked down
GGPPS-1 in vitro in U87 cells using shRNA targeting GGPPS-1. This shRNA downregulated GGPPS-1 protein expression by $50 \%$ at $48 \mathrm{~h}$ (Figure 4B). These cells survived the GGPP depletion without significant morphologic changes for 4-5 days. After this time point, however, U87 cells would detach and die with morphological changes very similar to those following statin treatment (Figure 4A). The downregulation of GGPPS-1 also induced cell autophagy, as shown by the LC3 western blot (Figure 4B). The cells were more sensitive to statin treatment as evidenced by the 10 -fold decrease in $\mathrm{IC}_{50}$ from 3.576 to $0.316 \mu \mathrm{M}$ (Figure 4C). More importantly, U87 cells with GGPPS-1 knockdown dramatically delayed tumour growth in nude mice (Figure 4D and E). The Ki67 staining showed that tumours with GGPPS-1 knockdown had fewer proliferating cells (Figure 4F). Our data demonstrate that the depletion of mevalonate pathway is an effective approach to inhibit tumour growth and proliferation, either by statins or by interference with critical enzymes in this pathway. 


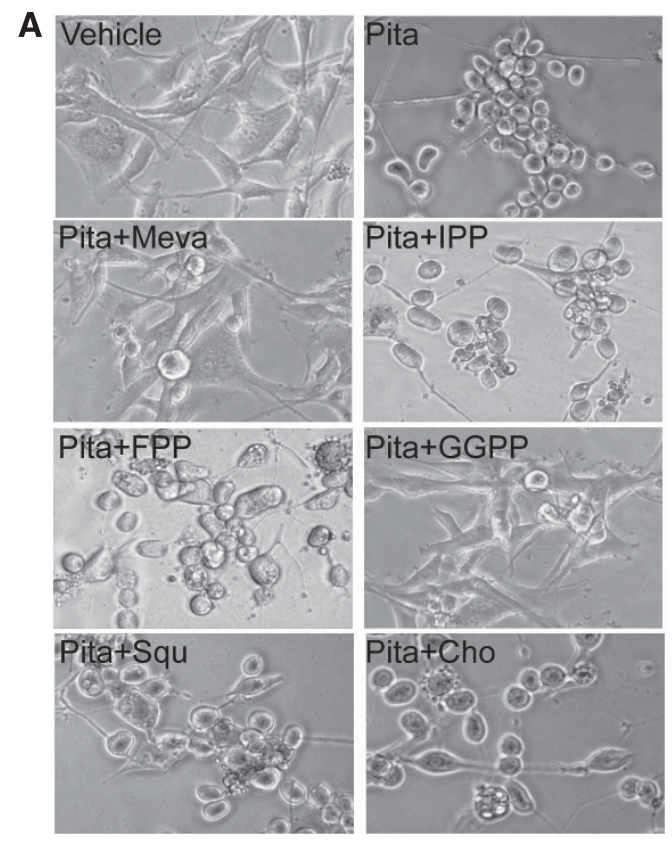

B
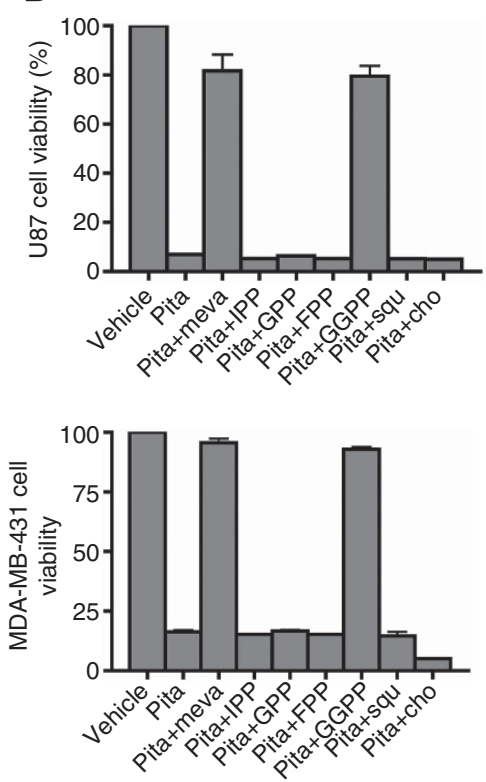

U87


Figure 3. Only mevalonate and GGPP can rescue tumour cells death after statin treatment. (A) The morphologic change of U87 and MDA-MB431 cell after pitavastatin with and without intermediate products of the mevalonate synthesis pathway. (B) Measurement of tumour cell viability after pitavastatin treatment and rescue also confirmed that mevalonolactone and GGPP can rescue cell death, but adding IPP, GPP, FPP, squalene, and cholesterol had little or no effect after pitavastatin treatment. After pitavastatin treatment, U87 cell viability decreased to $6.96 \% \pm 0.17 \%$ $(P<0.001)$, and on addition of mevalonolactone and GGPP, the cell viability rescued to $81.63 \% \pm 11.55 \%(P=0.11)$ and $79.57 \% \pm 7.17 \%$ $(P=0.039)$. For MDA-MB-431, after pitavastatin treatment, the cell viability decrease to $16.29 \% \pm 1.32 \%(P<0.001)$, but after adding mevalonolactone and GGPP, the cell viability rescued to $95.63 \% \pm 3.02 \%(P=0.13)$ and $94.24 \% \pm 1.45 \%(P=0.02)$. (C) GGPP also blocked the cell autophagy induced by pitavastatin as the GFPLC3 puncta forming also abolished after adding GGPP.

Statins inhibit tumour growth in animal models. We tested the effect of either pitavastatin or fluvastatin on GBM in xenograft mouse models. Pitavastatin significantly delayed subcutaneous U87 tumour growth as demonstrated by tumour size and tumour weight at a dose of $1 \mathrm{mg} \mathrm{kg}^{-1}$ per day i.p. (Figure $5 \mathrm{~A}$ and $\mathrm{B}$ ). However, a similar dose of fluvastatin was not as potent as pitavastatin; there was no significant difference between the U87 tumour treated with fluvastatin or PBS (vehicle). This result is consistent with the in vitro findings that the $\mathrm{IC}_{50}$ of fluvastatin was 3-5 times higher than pitavastatin. Ki67 staining also confirmed that U87 tumour after pitavastatin treatment had less proliferation compared with U87 tumour treated with fluvastatin or vehicle (Figure 5C). We also tested the effect of cerivastatin used at the same dose; this statin also delayed subcutaneous U87 tumour growth as demonstrated by tumour size and tumour weight (Supplementary Figure 11). However, the inhibitory effect of cerivastatin was lower than that of pitavastatin. After cerivastatin treatment, the average tumour size decreased by $50.2 \%$, but at the end point of the study with pitavastatin, tumour size decreased by $74.3 \%$. On the basis of these results, it may be useful to compare the in vivo effect of all statins using a higher dosage, such as $10-50 \mathrm{mg} \mathrm{kg}^{-1}$ per day to define the best statin that inhibits tumour growth.

Pitavastatin is currently approved for oral administration in humans; hence we tested whether orally administered pitavastatin has anticancer effect on U87 tumour growth. Using oral gavage, a higher dose of pitavastatin $\left(4-8 \mathrm{mg} \mathrm{kg}^{-1}\right.$ per day) in tumourbearing mice did not inhibit U87 tumour growth significantly although the mean tumour size was smaller and mean tumour weight was less than control (Figure 5D and E). Our results suggest that the use of statins for anticancer therapy may necessitate modification of the formulation and optimising the dose.

\section{DISCUSSION}

In the last two decades, the successful and widespread uses of statin drugs for hypercholesterolaemia have revealed their potential anticancer effects. Recently, a study in Danish population with a diagnosis of cancer between 1995 and 2007 found that the use of statins is associated with reduced cancer-related mortality, with a reduction of up to $15 \%$ for the 13 cancer types examined (Nielsen et al, 2012). Another population-based cohort study in Taiwan demonstrated that after HBV infection, the overall incidence rate for hepatocellular carcinoma rate was 310.4/100 000 person-year for nonusers, but for statin users the rate was dramatically lowered to $210.9,260,198.1$, and 158.7 with increasing doses of the statin (Tsan et al, 2012). These are the most recent examples from a wealth of data that support reduced cancer incidence, recurrence, and mortality with the use of statins (Boudreau et al, 2010). 
A

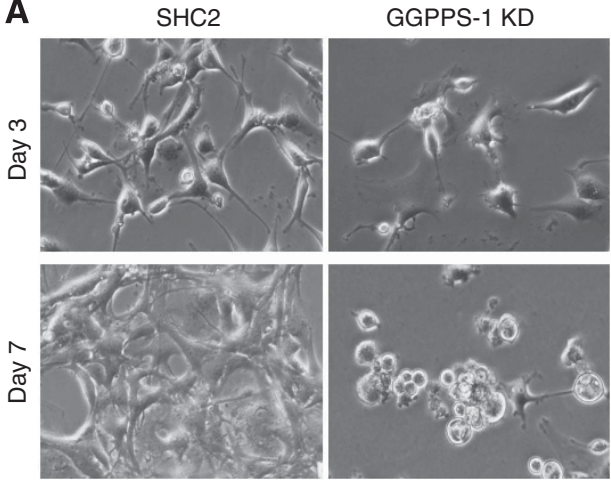

D

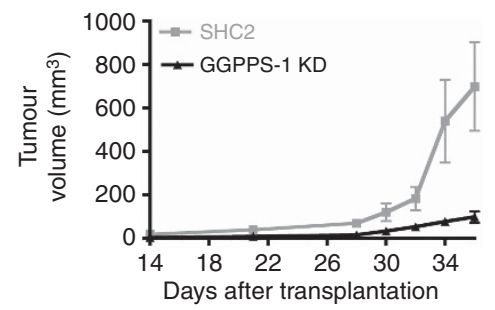

E
B



C



$\mathbf{F}$
ญ़
ஸे



Figure 4. Knockdown of GGPPS-1 gene in U87 cells induced autophagy and cell death in vitro and the tumour growth dramatically delayed in vivo. (A) GGPPS-1 knockdown induced U87 cell turn rounding, detachment, and death at day 5-7. (B) Western blotting of GGPPS-1 showed that knockdown of GGPPS-1 induced cellular autophagy signal as evidenced by increase in the LC3-II isoform. (C) The IC 50 of pitavastatin decreased from $3.6 \mu \mathrm{m}$ to $0.37 \mu \mathrm{m}$ after GGPPS-1 knockdown. (D) GGPPS-1 knockdown in U87 cells significantly slowed tumour growth when compared with control, as per measurement of tumour size. (E) Knockdown of GGPPS-1 slowed down U87 tumour growth cells as measured by tumour weight. The average tumour weight is $0.63 \mathrm{~g} \pm 0.43 \mathrm{~g}$ vs $0.12 \pm 0.1 \mathrm{~g}(P=0.018, \mathrm{n}=6)(\mathrm{F})$ The Ki67 staining showed that GGPPS-1 KD tumour have less proliferation cells.

The strong and consistent association of statins and lowered cancer risk has prompted 18 ongoing clinical trials in cancer (Clendening and Penn, 2012).

Statins are competitive inhibitors of HMGCR, which converts HMG-CoA to mevalonate. This is the rate-limiting step in cholesterol biosynthesis (Goldstein and Brown, 1990). Cholesterol is an essential component of cell membrane; the rapidly growing cancer tumour cells require a high uptake of extracellular cholesterol, but many patients with cancer have reduced plasma levels of cholesterol (Fiorenza et al, 2000; Benn et al, 2011). Therefore, statins can inhibit human cancer growth and thus reduce mortality by decreasing the locally synthesised or circulating cholesterol levels. In addition to cholesterol, HMGCR inhibition by statins also depletes several other important intermediates. Two such intermediates, FPP and GGPP modify many oncogenic proteins, including Ras and Rho GTPase for their malignant transforming activity (Jackson et al, 1990; Colditz et al, 2006). Another important product is dolichol, which is a carbohydrate donor during $N$-linked glycosylation of membranetargeted proteins (Schenk et al, 2001; Spiro, 2002). Statins deplete dolichol synthesis, block insulin and IGF receptor glycosylation, and impair tumour cell growth (Carlberg et al, 1996; Siddals et al, 2004). In a previous report, statins can impair the glycosylation of FLT3, a class III tyrosine kinase receptor, thus leading to a loss of surface expression and induction of cell death of FLT3 mutant acute myeloid leukaemia (Williams et al, 2012). Our previous study, also showed that pitavastatin depleted glycosylation of multidrug resistance protein 1 and enhanced irinotecan accumulation and then cell death in tumour cell (Jiang et al, 2014). The mevalonate pathway also supplies substrates to make isopentenyltRNA, haem-A and ubiquinone that are involved in the electron transport chain (Edwards and Ericsson, 1999). How these products are involved in tumour survival and proliferation is unclear.

In our studies, we found that knockdown of GGPPS-1 induced cell death and retarded tumour growth; our data strongly suggest that other enzymes in the mevalonate pathway may present novel cancer therapeutic targets (Dudakovic et al, 2010). Hohl and collaborators developed bisphosphonate inhibitors of GGPPS-1 that were highly effective in chronic myeloid leukaemia cells and MDA-MB-231 breast cancer cell lines (Dudakovic et al, 2008). However, these compounds still have very high $\mathrm{IC}_{50}$ and are not available commercially. Our data confirms that GGPP is a critical isoprenoid for tumour cell viability and proliferation. In addition, GGPPS-1 knockdown dramatically inhibits GBM tumour growth in vivo.

Other than statins, HMGCR can be phosphorylated and inactivated by 5' adenosine monophosphate-activated protein kinase (AMPK; Hayashi et al, 1998). AMPK is an enzyme that has an essential role in cellular energy homeostasis. AICAR (5-amino-1- $\beta$-D-ribofuranosyl-imidazole-4-carboxamide), also known as $\mathrm{ZMP}$, is an analogue of AMP that is capable of stimulating AMPK activity (Guo et al, 2009). AICAR is being used clinically to 
A

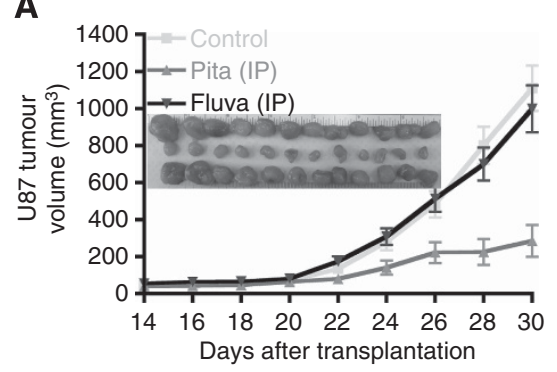



C
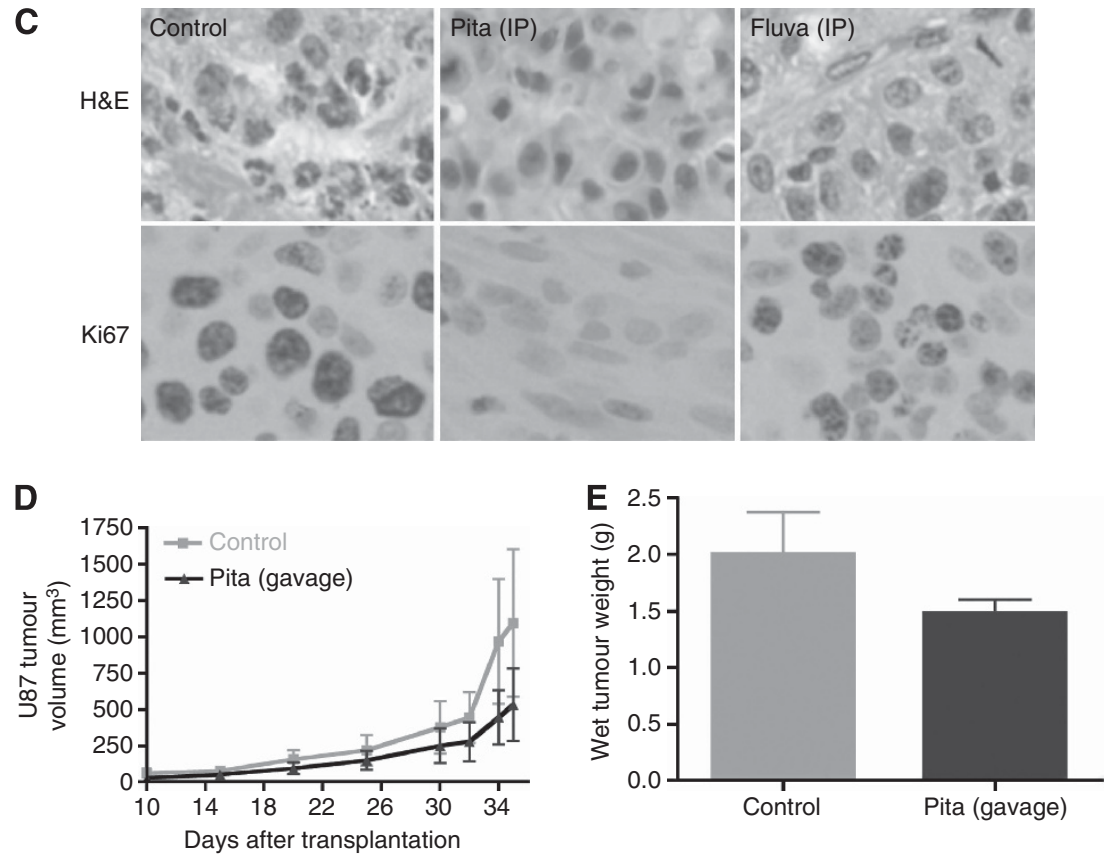

Figure 5. Statin delayed GBM tumour growth in mouse model. (A) A total of $1 \mathrm{mg} \mathrm{kg}^{-1}$ pitavastatin (Pita) by i.p. injection slowed U87 tumour growth when compared with control; $1 \mathrm{mg} \mathrm{kg}^{-1}$ of fluvastatin (Fluva) did not show inhibition of tumour growth. (B) U87 tumour weight after pitavastatin treatment was much significantly lower than control but fluvastatin-treated tumour had no effect on tumour weight when compared with the control group. The average tumour weight is $0.93 \pm 0.25 \mathrm{~g}, 0.21 \pm 0.12 \mathrm{~g}$ and $0.89 \pm 0.22 \mathrm{~g}$ for control, pita and fluva group, for control and pita group $P<0.001$, for control and flu group $P=0.36$. (C) Fewer proliferative cells were detected in the tumour after pitavastatin treatment according to the Ki67 staining results but not in fluvastatin group. (D) Gavage higher dose of 4-8 $\mathrm{mg} \mathrm{kg}^{-1}$ pitavastatin slightly delayed tumour growth but the difference was not statistically significant. (E) U87 tumour weight did not show statistically significant difference from control group after gavage pitavastatin treatment: average tumour weight is $2.0 \pm 1.2 \mathrm{~g}$ vs $1.5 \pm 0.4 \mathrm{~g}(P=0.12, \mathrm{n}=12)$.

treat and protect against cardiac ischaemic injury. AICAR is highly soluble with little cellular toxicity and easily crosses the BBB. As our data in Supplementary Figure S10 demonstrate, co-existing AICAR can decease the $\mathrm{IC}_{50}$ of pitavastatin from 2.375 to $0.4625 \mu \mathrm{M}$, indicating that this drug combination may be a good candidate for further research and clinical studies (Vakana et al, 2011).

Although clear anticancer effects have been observed for all statins, the existing data suggest certain differences in the antitumor effects of individual statins (Gbelcova et al, 2008). Statins have marked disparities in pharmacokinetics, potency, and therapeutic efficacy. Comparative studies have reported significant differences in the rates of decrease of LDL-cholesterol, triglycerides, and phospholipid levels (Bergheanu et al, 2008; Leszczynska et al, 2009). In addition, statins are not primarily designed for cancer therapy, so the anticancer effects need to be carefully evaluated and compared before one can optimise the efficacy of statins as anticancer agents. Our work here demonstrates that the best three statins against human cancer cells growth in vitro are cerivastatin, pitavastatin, and fluvastatin in all three types of cancer cells tested: serum-grown breast and GBM cell lines and patientderived GBM stem cell-like primary cells grown as neurospheres.
Our study investigated the potency of pitavastatin and fluvastatin. Consistent with in vitro results, we found that pitavastatin is better than fluvastatin in vivo. However, the same dose cerivastatin is less potent than pitavastaitn, this is inconsistent with the in vitro results. Statins differ in lipophilicity, rate and efficacy of absorption, metabolism, and excretion, as well as such properties as diffusion through the blood-brain barrier or circulation half-life; these properties may determine the efficacy to cancer therapy in vivo. We also confirmed that IP injection improved the efficacy of statins compared with oral administration. Importantly, our results indicate that additional in vivo investigation of the anticancer efficacy of statins is necessary. Moreover, as we show here, other enzymes in the cholesterol synthesis pathway are also potential targets for cancer therapy.

Interestingly, the in vitro $\mathrm{IC}_{50}$ is achievable for cerivastatin and pitavastatin in humans at standard doses of $0.2-0.4 \mathrm{mg}$ per day and 1-4 mg per day, respectively (Kajinami et al, 2000). These data suggest that statin drug doses targeting tumour cells specifically on the mevalonate pathway are the same as those used to control hypercholesterolaemia. Autophagy is a cellular catabolic degradation response to starvation or stress by recycling organelles and cytoplasmic macromolecules (Levine and Klionsky, 2004; 
Eskelinen, 2005). Paradoxically, autophagy is the key to tumour suppression and cancer cell survival (Levine, 2007; Mathew et al, 2007). Understanding the link between statin and cell death in the form of autophagy or apoptosis may help develop new approaches to cancer therapy. Whether the autophagy induced by statins contributes to the statin-induced tumour growth inhibition is still unclear. Further, the lowering of $\mathrm{IC}_{50}$ following longer treatment durations may explain how the effect of some less potent statins in our screening could be enhanced.

These data indicate that the accumulation of statins in the tumour and the maintenance of higher statin concentrations in plasma and in tumour for longer durations could make critical contribution to statins' anticancer effect.

\section{ACKNOWLEDGEMENTS}

This work was supported in part by grants from NIH (NIH 3P30CA023100-25S8) and Tuttleman Family Foundation to SK. We thank Gabi Gomez for technical assistance in the laboratory.

\section{CONFLICT OF INTEREST}

The following authors are employed by Cellworks, Inc.: Shweta Kapoor, Krithika Shetty, Ashish Aggrawal, Shireen Vali, Taher Abbasi. The remaining authors declare no conflict of interest.

\section{AUTHOR CONTRIBUTIONS}

PJ, SK designed the research, PJ, TA wrote the manuscript, SK, SCP, SP revised the manuscript, PJ, RM, YC, NN, ISB, VF, SP, DT, $\mathrm{XC}, \mathrm{SK}, \mathrm{KS}, \mathrm{AA}, \mathrm{SV}$ performed the experiments.

\section{REFERENCES}

Alberts AW, Chen J, Kuron G, Hunt V, Huff J, Hoffman C, Rothrock J, Lopez M, Joshua H, Harris E, Patchett A, Monaghan R, Currie S, Stapley E, Albers-Schonberg G, Hensens O, Hirshfield J, Hoogsteen K, Liesch J, Springer J (1980) Mevinolin: a highly potent competitive inhibitor of hydroxymethylglutaryl-coenzyme A reductase and a cholesterol-lowering agent. Proc Natl Acad Sci USA 77: 3957-3961.

Benn M, Tybjaerg-Hansen A, Stender S, Frikke-Schmidt R, Nordestgaard BG (2011) Low-density lipoprotein cholesterol and the risk of cancer: a mendelian randomization study. J Natl Cancer Inst 103: 508-519.

Bergheanu SC, Reijmers T, Zwinderman AH, Bobeldijk I, Ramaker R, Liem AH, van der Greef J, Hankemeier T, Jukema JW (2008) Lipidomic approach to evaluate rosuvastatin and atorvastatin at various dosages: investigating differential effects among statins. Curr Med Res Opin 24: 2477-2487.

Boudreau DM, Yu O, Johnson J (2010) Statin use and cancer risk: a comprehensive review. Expert Opin Drug Saf 9: 603-621.

Brown MS, Goldstein JL (1986) A receptor-mediated pathway for cholesterol homeostasis. Science 232: 34-47.

Carlberg M, Dricu A, Blegen H, Wang M, Hjertman M, Zickert P, Hoog A, Larsson O (1996) Mevalonic acid is limiting for N-linked glycosylation and translocation of the insulin-like growth factor-1 receptor to the cell surface. Evidence for a new link between 3-hydroxy-3-methylglutarylcoenzyme a reductase and cell growth. J Biol Chem 271: 17453-17462.

Clendening JW, Penn LZ (2012) Targeting tumor cell metabolism with statins. Oncogene 31: 4967-4978.

Colditz GA, Sellers TA, Trapido E (2006) Epidemiology - identifying the causes and preventability of cancer? Nat Rev Cancer 6: 75-83.

Dimitroulakos J, Nohynek D, Backway KL, Hedley DW, Yeger H, Freedman MH, Minden MD, Penn LZ (1999) Increased sensitivity of acute myeloid leukemias to lovastatin-induced apoptosis: A potential therapeutic approach. Blood 93: 1308-1318.
Dudakovic A, Tong H, Hohl RJ (2010) Geranylgeranyl diphosphate depletion inhibits breast cancer cell migration. Invest New Drugs 29: 912-920.

Dudakovic A, Wiemer AJ, Lamb KM, Vonnahme LA, Dietz SE, Hohl RJ (2008) Inhibition of geranylgeranyl diphosphate synthase induces apoptosis through multiple mechanisms and displays synergy with inhibition of other isoprenoid biosynthetic enzymes. J Pharmacol Exp Ther 324: $1028-1036$.

Edwards PA, Ericsson J (1999) Sterols and isoprenoids: signaling molecules derived from the cholesterol biosynthetic pathway. Annu Rev Biochem 68: $157-185$.

Endo A (2004) The origin of the statins. Atheroscler Suppl 5: 125-130.

Endo A, Kuroda M, Tanzawa K (1976) Competitive inhibition of 3-hydroxy3-methylglutaryl coenzyme A reductase by ML-236A and ML-236B fungal metabolites, having hypocholesterolemic activity. FEBS Lett 72: 323-326.

Eskelinen EL (2005) Maturation of autophagic vacuoles in Mammalian cells. Autophagy 1: 1-10.

Fiorenza AM, Branchi A, Sommariva D (2000) Serum lipoprotein profile in patients with cancer. A comparison with non-cancer subjects. Int J Clin Lab Res 30: 141-145.

Furberg CD, Pitt B (2001) Withdrawal of cerivastatin from the world market. Curr Control Trials Cardiovasc Med 2: 205-207.

Gbelcova H, Lenicek M, Zelenka J, Knejzlik Z, Dvorakova G, Zadinova M, Pouckova P, Kudla M, Balaz P, Ruml T, Vitek L (2008) Differences in antitumor effects of various statins on human pancreatic cancer. Int J Cancer 122: 1214-1221.

Goldstein JL, Brown MS (1990) Regulation of the mevalonate pathway. Nature 343: 425-430.

Guo D, Hildebrandt IJ, Prins RM, Soto H, Mazzotta MM, Dang J, Czernin J, Shyy JY, Watson AD, Phelps M, Radu CG, Cloughesy TF, Mischel PS (2009) The AMPK agonist AICAR inhibits the growth of EGFRvIIIexpressing glioblastomas by inhibiting lipogenesis. Proc Natl Acad Sci USA 106: $12932-12937$.

Hayashi T, Hirshman MF, Kurth EJ, Winder WW, Goodyear LJ (1998) Evidence for 5' AMP-activated protein kinase mediation of the effect of muscle contraction on glucose transport. Diabetes 47: 1369-1373.

Jackson JH, Cochrane CG, Bourne JR, Solski PA, Buss JE, Der CJ (1990) Farnesol modification of Kirsten-ras exon $4 \mathrm{~B}$ protein is essential for transformation. Proc Natl Acad Sci USA 87: 3042-3046.

Jakobisiak M, Bruno S, Skierski JS, Darzynkiewicz Z (1991) Cell cycle-specific effects of lovastatin. Proc Natl Acad Sci USA 88: 3628-3632.

Jiang P, Mukthavaram R, Chao Y, Bharati IS, Fogal V, Pastorino S, Cong X, Nomura N, Gallagher M, Abbasi T, Vali S, Pingle SC, Makale M, Kesari S (2014) Novel anti-glioblastoma agents and therapeutic combinations identified from a collection of FDA approved drugs. J Transl Med 12: 13.

Jones KD, Couldwell WT, Hinton DR, Su Y, He S, Anker L, Law RE (1994) Lovastatin induces growth inhibition and apoptosis in human malignant glioma cells. Biochem Biophys Res Commun 205: 1681-1687.

Kajinami K, Mabuchi H, Saito Y (2000) NK-104: a novel synthetic HMG-CoA reductase inhibitor. Expert Opin Investig Drugs 9: 2653-2661.

Keyomarsi K, Sandoval L, Band V, Pardee AB (1991) Synchronization of tumor and normal cells from G1 to multiple cell cycles by lovastatin. Cancer Res 51: 3602-3609.

Leszczynska A, Burzynska B, Plochocka D, Kaminska J, Zimnicka M, Kania M, Kiliszek M, Wysocka-Kapcinska M, Danikiewicz W, Szkopinska A (2009) Investigating the effects of statins on cellular lipid metabolism using a yeast expression system. PLoS One 4: e8499.

Levine B (2007) Cell biology: autophagy and cancer. Nature 446: 745-747.

Levine B, Klionsky DJ (2004) Development by self-digestion: molecular mechanisms and biological functions of autophagy. Dev Cell 6: 463-477.

Mathew R, Karantza-Wadsworth V, White E (2007) Role of autophagy in cancer. Nat Rev Cancer 7: 961-967.

Nielsen SF, Nordestgaard BG, Bojesen SE (2012) Statin use and reduced cancer-related mortality. N Engl J Med 367: 1792-1802.

Pingle SC, Sultana Z, Pastorino S, Jiang PF, Mukthavaram R, Chao Y, Bharati IS, Nomura N, Makale M, Abbasi T, Kapoor S, Kumar A, Usmani S, Agrawal A, Vali S, Kesari S (2014) In Silico deterministic modeling predicts drug sensitivity of patient-derived cancer cells. J Transl Med 12: 128.

Sane KM, Mynderse M, Lalonde DT, Dean IS, Wojtkowiak JW, Fouad F, Borch RF, Reiners Jr JJ, Gibbs RA, Mattingly RR (2010) A novel geranylgeranyl transferase inhibitor in combination with lovastatin 
inhibits proliferation and induces autophagy in STS-26T MPNST cells. J Pharmacol Exp Ther 333: 23-33.

Schenk B, Fernandez F, Waechter CJ (2001) The inside and outside of dolichyl phosphate biosynthesis and recycling in the endoplasmic reticulum. Glycobiology 11: 61R-70R.

Siddals KW, Marshman E, Westwood M, Gibson JM (2004) Abrogation of insulin-like growth factor-I (IGF-I) and insulin action by mevalonic acid depletion: synergy between protein prenylation and receptor glycosylation pathways. J Biol Chem 279: 38353-38359.

Spiro RG (2002) Protein glycosylation: nature, distribution, enzymatic formation, and disease implications of glycopeptide bonds. Glycobiology 12: $43 \mathrm{R}-56 \mathrm{R}$.

Thurnher M, Nussbaumer O, Gruenbacher G (2012) Novel aspects of mevalonate pathway inhibitors as antitumor agents. Clin Cancer Res 18: 3524-3531.

Tobert JA (2003) Lovastatin and beyond: the history of the HMG-CoA reductase inhibitors. Nat Rev Drug Discov 2: 517-526.
Tsan YT, Lee CH, Wang JD, Chen PC (2012) Statins and the risk of hepatocellular carcinoma in patients with hepatitis B virus infection. J Clin Oncol 30: 623-630.

Vakana E, Altman JK, Platanias LC (2011) Targeting AMPK in the treatment of malignancies. J Cell Biochem 113: 404-409.

Williams AB, Li L, Nguyen B, Brown P, Levis M, Small D (2012) Fluvastatin inhibits FLT3 glycosylation in human and murine cells and prolongs survival of mice with FLT3/ITD leukemia. Blood 120: 3069-3079.

Wong WW, Dimitroulakos J, Minden MD, Penn LZ (2002) HMG-CoA reductase inhibitors and the malignant cell: the statin family of drugs as triggers of tumor-specific apoptosis. Leukaemia 16: 508-519.

This work is published under the standard license to publish agreement. After 12 months the work will become freely available and the license terms will switch to a Creative Commons AttributionNonCommercial-Share Alike 3.0 Unported License.

Supplementary Information accompanies this paper on British Journal of Cancer website (http://www.nature.com/bjc) 